\title{
Commentaire
}

\section{Constituer un ensemble de données probantes au service d'une action soutenue en santé publique en réponse à la crise des opioïdes au Canada}

\author{
Theresa Tam, BMBS, FRCPC
}

Diffuser cet article sur Twitter

L'épidémie de surdoses liées aux opioïdes constitue la principale crise en santé publique depuis deux ans, ayant nécessité une réponse collective de tous les ordres de gouvernement, en collaboration avec les répondants de première ligne et divers autres partenaires. On estime malheureusement à plus de 4000 le nombre de décès apparemment liés aux opioïdes en 2017 au Canada, ce qui dépasse les quelque 3000 décès déclarés pour 2016. La crise touche les Canadiens de tous les groupes socioéconomiques, dans les collectivités urbaines et rurales comme dans les petits centres de l'ensemble du pays ${ }^{1}$. Si l'Ouest a été plus durement touché jusqu'ici, nos données les plus récentes font ressortir une tendance à la hausse dans d'autres régions du pays. Contrer cette crise de santé publique complexe constitue une priorité pour les autorités responsables de la santé publique de tous les ordres de gouvernement. Il est crucial pour cela de disposer d'un ensemble solide de données probantes en soutien aux efforts que nous déployons pour agir dans l'urgence et de manière ciblée afin de prévenir les surdoses mortelles et d'éliminer les causes sous-jacentes de la consommation problématique de substances.

L’Agence de la santé publique du Canada (ASPC) joue un rôle essentiel dans la réponse coordonnée du gouvernement du Canada à la crise des opiö̈des, dirigée par l'Équipe d'intervention en matière d'opioïdes de Santé Canada. Cette réponse mise sur la collaboration, par le biais d'activités d'intervention d'urgence en santé publique qui s'articulent autour de quatre piliers : prévention, traitement, réduction des méfaits et application des règlements. Soutenant ces quatre piliers, un ensemble solide de données probantes permet de mieux dégager les tendances, d'éclairer la prise de décisions, de cibler les interventions et d'en mesurer l'impact. À l'heure actuelle, l'ASPC agit comme chef de file dans la réalisation de deux études épidémiologiques et intervient également pour consolider et élargir les activités de surveillance des méfaits liés aux opioïdes, afin d'approfondir notre compréhension des circonstances qui entourent les surdoses mortelles d'opioïdes au Canada. Ces initiatives vont combler diverses lacunes dans la compréhension des facteurs de risque et des causes des surdoses mortelles d'opioïdes, ce qui va permettre de faciliter les décisions alimentant les politiques publiques et d'élaborer ainsi que de mieux cibler les interventions. L'ASPC fournit également un soutien technique aux provinces et aux territoires en leur affectant localement des agents en santé publique afin d'assurer un soutien dans la collecte des données et dans l'amélioration de l'infrastructure de surveillance. L'ASPC prévoit également de travailler avec ses partenaires des collectivités des Premières Nations, des Inuits et des Métis afin de définir des stratégies de surveillance aptes à combler les principales lacunes qui nuisent à notre compréhension de l'impact de la crise sur les Autochtones du Canada.

Je suis pleinement consciente du fait qu'aucun ministère ou gouvernement ne peut s'attaquer efficacement à cette épidémie en faisant cavalier seul. La collaboration intergouvernementale et intersectorielle est indispensable. L'ASPC travaille ainsi étroitement avec les autorités sanitaires provinciales et territoriales par l'entremise du Comité consultatif spécial sur l'épidémie de surdoses d'opioïdes, que je copréside avec le $\mathrm{D}^{\mathrm{r}}$ Robert Strang, médecin hygiéniste en chef de la Nouvelle-Écosse.
Depuis sa création en décembre 2016, le Comité consultatif spécial favorise la collaboration et la mise en commun d'information entre les différentes instances à l'appui des efforts de réduction des méfaits, d'amélioration de la surveillance et d'élaboration de stratégies de prévention et de traitement.

Ce numéro spécial de Promotion de la santé et prévention des maladies chroniques au Canada : Recherche, politiques et pratiques est consacré à la crise des opioïdes. Par cette publication, nous souhaitons faire progresser notre compréhension collective de l'épidémie qui sévit au Canada, en examinant les données disponibles afin de quantifier le fardeau que cette crise constitue pour la société, en décrivant la manière dont les méfaits sont répartis ainsi que les facteurs de risque au sein de la population et les tendances au fil du temps, en explorant le contexte et les circonstances ayant mené à la crise et en faisant le point sur les pistes les plus prometteuses à explorer.

Deux des articles de ce numéro présentent une estimation du fardeau des méfaits liés aux opioïdes, celui de Ye et ses collaborateurs $^{2}$ et celui d'Orpana et ses collaborateurs $^{3}$. L'article de Ye et ses collaborateurs ${ }^{2}$ porte sur la contribution des surdoses mortelles de drogues à l'espérance de vie en Colombie-Britannique. Les auteurs calculent qu'entre 2014 et 2016, l'espérance de vie à la naissance a reculé de 0,38 an, et que le tiers de ce recul est attribuable aux surdoses mortelles (d'opioïdes, principalement). Orpana et ses collaborateurs ${ }^{3}$ traitent des taux de morbidité et de mortalité liés aux opiö̈des et, d'après leurs estimations, entre 1990 et 2014, le taux 
normalisé selon l'âge d'années potentielles de vie perdues (APVP) liées aux opioïdes s'est accru de plus de $142 \%$ au Canada, alors qu'il a reculé de $10 \%$ à l'échelle mondiale.

O'Connor et ses collaborateurs ${ }^{4}$ se penchent quant à eux sur les tendances nationales et régionales des hospitalisations attribuables aux intoxications aux opioïdes. D'après leurs estimations, ces hospitalisations ont, en dix ans (2007-2008 à 2016-2017) progressé de plus de $50 \%$ au Canada. Ils ont également constaté qu'entre 2012-2013 et 2016-2017, le taux normalisé selon l'âge des visites au service des urgences liées à des intoxications aux opioïdes s'est accru de plus de $100 \%$ en Alberta et de près de $50 \%$ en Ontario.

Dans leurs articles, Belzak et Halverson ${ }^{5}$ ainsi que Bozat-Emre et ses collaborateurs ${ }^{6}$ se penchent sur les facteurs de risque et les caractéristiques sociodémographiques associés aux méfaits liés aux opiö̈des. Belzak et Halverson ${ }^{5}$ offrent un survol national de ce que l'on sait sur l'épidémie et s'intéressent aux facteurs de risque potentiels ainsi qu'aux variations observées en fonction de l'âge, du sexe et de l'ethnicité. Bozat-Emre et ses collaborateurs ${ }^{6}$ utilisent des données du programme de naloxone à emporter du gouvernement du Manitoba afin de cerner les caractéristiques sociodémographiques des utilisateurs de ce programme.

Guan et ses collaborateurs ${ }^{7}$ s'intéressent à la question des ordonnances d'opioïdes et font une estimation de l'incidence qu'a eu le retrait, en 2016, des opioïdes très puissants de la liste de médicaments du régime public de l'Ontario sur les habitudes de prescription des médecins.

Enfin, Tibebu et ses collaborateurs ${ }^{8}$ se demandent en quoi les données des médias sociaux peuvent nous aider à comprendre les perceptions et les opinions du public concernant la consommation d'opioïdes et la réponse des institutions publiques.

Les résultats de recherche présentés dans ces articles contribuent à réaliser notre objectif, qui est de constituer un ensemble solide de données probantes apte à soutenir la planification et la prise de décisions au service d'une action en santé publique la plus pertinente possible.

Un second numéro spécial consacré aux opioïdes sera publié en septembre 2018. Il y sera question des facteurs de risque dans la Cohorte provinciale de la ColombieBritannique sur les surdoses, du rôle joué par les opioïdes dans les suicides en Alberta ainsi que des intoxications liées aux opioïdes chez les jeunes, de l'incidence des changements apportés à la politique de prescription d'opioïdes en Nouvelle-Écosse et enfin d'une approche stratégique visant à régler la crise en Ontario.

La communauté en santé publique que nous formons doit poursuivre ses efforts de sensibilisation aux risques associés à la consommation problématique d'opioïdes, en soulignant que cette crise continue à toucher des Canadiens de tous les âges et de tous les milieux. Pour y parvenir, il est crucial de réduire la stigmatisation liée à la consommation problématique de substances, car elle pose un obstacle de taille aux efforts déployés pour réduire les méfaits, même lorsque les interventions sont bien conçues. La collaboration des Canadiens qui ont connu ou connaissent des troubles liés à la consommation d'opioïdes, en particulier les jeunes et les Autochtones, se révèle prioritaire.

Les connaissances de première main sont utiles à la fois pour les décideurs, les chercheurs, les médecins et les praticiens en santé publique. Maintenir une action concertée et soutenue est le seul moyen de renverser la trajectoire actuelle de l'épidémie. En continuant à travailler dans toutes les disciplines, tous les secteurs de la société et à tous les ordres de gouvernement, nous allons pouvoir trouver des solutions efficaces et durables pour éviter ces pertes de vie tragiques et pour protéger la santé de la population canadienne.

J'aimerais profiter de cette occasion pour souligner le travail des nombreuses personnes et des nombreux organismes qui contribuent à la résolution de cette crise. Je salue vos efforts soutenus et je vous encourage à les poursuivre afin que nous réussissions à réduire la mortalité et à prévenir les graves méfaits associés aux troubles de consommation d'opioïdes à l'échelle du pays. Je suis convaincue que les articles présentés dans ce numéro vont enrichir grandement l'ensemble de données dont nous avons besoin pour orienter et soutenir nos efforts collectifs.

\section{$\mathrm{D}^{\text {re }}$ Theresa Tam Administratrice en chef de la santé publique du Canada}

\section{Références}

1. Comité consultatif spécial sur l'épidémie de surdoses d’opioïdes. Rapport national : Décès apparemment liés à la consommation d'opioïdes (décembre 2017) [Internet]. Ottawa (ON): Agence de la santé publique du Canada; 2017. En ligne à : https:// www.canada.ca/fr/sante-publique /services/publications/vie-saine/deces -apparemment-lies-consommation -opioides-rapport-2016-2017-decembre .html

2. Ye X, Sutherland J, Henry B, et al. Aperçu - Impact des décès par surdose de drogue sur l'espérance de vie à la naissance en Colombie-Britannique. Promotion de la santé et prévention des maladies chroniques au Canada. 2018;38(6):282-286.

3. Orpana HM, Lang JL, Baxi M, et al. Tendances canadiennes en matière de mortalité liée aux opioïdes et d'invalidité découlant d'un trouble de consommation d'opioïdes, à la lumière de l'Étude sur la charge mondiale de morbidité (1990-2014). Promotion de la santé et prévention des maladies chroniques au Canada. 2018;38(6): 267-277.

4. O’Connor S, Grywacheski V, Louie K. Aperçu - Hospitalisations et visites à l'urgence en raison d'un empoisonnement aux opioïdes au Canada. Promotion de la santé et prévention des maladies chroniques au Canada. 2018;38(6):278-281.

5. Belzak L, Halverson J. La crise des opioïdes au Canada : une perspective nationale. Promotion de la santé et prévention des maladies chroniques au Canada. 2018;38(6):255-266.

6. Bozat-Emre S, Marshall SG, Zhong C, et al. Aperçu - Leçons tirées du lancement du programme de naloxone à emporter à domicile du Manitoba. Promotion de la santé et prévention des maladies chroniques au Canada. 2018;38(6):287-290.

7. Guan Q, Khuu W, Martins D, et al. Évaluation des premiers effets du retrait des opioïdes à forte concentration sur les schémas de prescription en Ontario. Promotion de la santé et prévention des maladies chroniques au Canada. 2018;38(6):291-298. 
8. Tibebu S, Chang VC, Drouin C, et al. Aperçu - Que révèlent les médias sociaux au sujet de la crise des opioïdes au Canada? Promotion de la santé et prévention des maladies chroniques au Canada. 2018;38(6):299-305. 